VOL. 65 (2002) [521-526]

\title{
THE COMPOSITIONAL INVERSE OF A CLASS OF PERMUTATION POLYNOMIALS OVER A FINITE FIELD
}

\author{
Robert S. Coulter and Marie Henderson
}

\begin{abstract}
A new class of bilinear permutation polynomials was recently identified. In this note we determine the class of permutation polynomials which represents the functional inverse of the bilinear class.
\end{abstract}

\section{Introduction AND MAIN RESUlt}

Throughout $\mathbb{F}_{q}$ denotes the finite field of $q=p^{e}$ elements for some prime $p$ and positive integer $e$ with $\mathbb{F}_{q}[X]$ representing the ring of polynomials in the indeterminate $X$ over $\mathbb{F}_{q}$. For polynomials $f, g \in \mathbb{F}_{q}[X]$, we write $f \circ g=f(g(X))$ for the functional composition of $f$ with $g$. A permutation polynomial over $\mathbb{F}_{q}$ is a polynomial which, under evaluation, induces a permutation of the elements of $\mathbb{F}_{q}$. Clearly, permutation polynomials are the only polynomials which have a (functional) inverse with respect to composition, id est for a permutation polynomial $f \in \mathbb{F}_{q}[X]$ there exists (a unique) $f^{-1} \in \mathbb{F}_{q}$ such that $f\left(f^{-1}(X)\right) \equiv f^{-1}(f(X)) \equiv X \bmod \left(X^{q}-X\right)$. We call $f^{-1}$ the compositional inverse of $f$ (or vice versa).

The problem of discovering new classes of permutation polynomials is non-trivial and has generated much interest, see the surveys and open problems given in $[3,4,6]$. Discovering classes where the inverse polynomials can also be described seems to be even more difficult: there are very few known classes of permutation polynomials for which their compositional inverses are also known. To the authors knowledge, the classes with explicit formulae for inverses are:

(1) The linear polynomials: $X+a$ where $a \in \mathbb{F}_{q}$ is trivially a permutation polynomial of $\mathbb{F}_{q}$ with the inverse polynomial being $X-a$.

(2) The monomials: $X^{n}$ is a permutation polynomial over $\mathbb{F}_{q}$ if and only if $(n, q-1)=1$. In such cases, the compositional inverse of $X^{n}$ is obviously the monomial $X^{m}$ where $n m \equiv 1 \bmod (q-1)$.

Received 10th December, 2001

This research was supported by the Australian Research Council and the Information Security Research Centre at Queensland University of Technology.

Copyright Clearance Centre, Inc. Serial-fee code: 0004-9727/02 \$A2,00+0.00. 
(3) The Dickson polynomials of the 1st kind: $D_{n}(X, a)$ is a permutation polynomial over $\mathbb{F}_{q}$ if and only if $\left(n, q^{2}-1\right)=1$, see [5, Chapter 3]. In such cases, for $a \in\{0, \pm 1\}$, the compositional inverse of $D_{n}(X, a)$ is $D_{m}(X, a)$ where $n m \equiv 1 \bmod \left(q^{2}-1\right)$, see [5, Chapter 3].

We note that there are classes for which inverses can be determined (for example linearised and sub-linearised polynomials) but that no explicit formulas for the inverses are known.

Recently, a new class of permutation polynomials was introduced in [1]. Here we give a description for the compositional inverse of this class of permutation polynomials.

THEOREM 1. Let $q=2^{k}$ for some integer $k$. Let $n$ be an odd positive integer and set $Q=q^{n}$. Denote the trace mapping from $\mathbb{F}_{Q}$ to $\mathbb{F}_{q}$ by

$$
\operatorname{Tr}(X)=X+X^{q}+\cdots+X^{q^{n-1}} .
$$

For any $\alpha \in \mathbb{F}_{q} \backslash\{0,1\}$, the polynomial

$$
f_{\alpha}(X)=X \operatorname{Tr}(X)+(\alpha+1) X^{2}
$$

is a permutation polynomial over $\mathbb{F}_{Q}$. For $\alpha$ as above and for any integer $i$ satisfying $1 \leqslant i \leqslant k-1$, define

$$
C_{i}=\frac{\alpha^{2^{k-1}+2^{k-1-i}-1}+1}{\alpha+1} .
$$

Set

$$
A_{\alpha}(X)=C_{k-1}\left(X^{2^{n k-1}}+\alpha^{2^{k-1}-1} \operatorname{Tr}(X)^{2^{k-1}}\right)
$$

and

$$
B_{\alpha}(X)=\sum_{i=1}^{k-1} C_{i} \operatorname{Tr}(X)^{2^{k-1}-2^{k-1-i}}\left(\sum_{j=1}^{(n-1) / 2}\left(X \operatorname{Tr}(X)+X^{2}\right)^{2^{2 j k-2-i}}\right) .
$$

The polynomial $g_{\alpha}=A_{\alpha}+B_{\alpha}$ is the compositional inverse of $f_{\alpha}$ over $\mathbb{F}_{Q}$.

The polynomials $f_{\alpha}$ were shown to be permutation polynomials in [1]. From Theorem 1 we have the following obvious corollary.

Corollary 2. For $\alpha \in \mathbb{F}_{q} \backslash\{0,1\}$, the polynomials $g_{\alpha}$, as defined in Theorem 1 , are permutation polynomials over $\mathbb{F}_{Q}$

\section{The Proof of Theorem 1}

Our attention from this point is directed to establishing the remaining statements of Theorem 1 , which is to show that $g_{\alpha}$ is the compositional inverse of $f_{\alpha}$. Our proof involves establishing a set of sequential propositions, basically involving closer examination of $f_{\alpha} \circ g_{\alpha}$, primarily in terms of the two polynomials $A_{\alpha}$ and $B_{\alpha}$. For $n$ odd, we have $\operatorname{Tr}(\operatorname{Tr}(x))=\operatorname{Tr}(x)$. This identity is used many times in the following propositions. We begin by collecting some useful identities. 
Proposition 3. For $A_{\alpha}, B_{\alpha} \in \mathbb{F}_{Q}[X]$ and $C_{i}$ as defined in Theorem 1 we have

(i) $C_{i}^{2}=\left(\alpha^{\left(2^{k-i}-1\right)}+1\right) /\left(\alpha^{2}+1\right)$,

(ii) $A_{\alpha}^{2}(X) \equiv C_{k-1}^{2}\left(X+\alpha^{-1} \operatorname{Tr}(X)\right) \bmod \left(X^{Q}+X\right)$,

(iii) $\operatorname{Tr}\left(A_{\alpha}\right) \equiv \alpha^{2^{k-1}-1} \operatorname{Tr}(X)^{2^{k-1}} \bmod \left(X^{Q},+X\right)$, and

(iv) $\operatorname{Tr}\left(B_{\alpha}\right) \equiv 0 \bmod \left(X^{Q}+X\right)$.

Proof: (i) Squaring $C_{i}$ we obtain the identity:

$$
C_{i}^{2}=\frac{\alpha^{2^{k}+2^{k-i}-2}+1}{\alpha^{2}+1}=\frac{\alpha^{2^{k-i}-1}+1}{\alpha^{2}+1} .
$$

(ii) Squaring $A_{\alpha}(X)$ gives $C_{k-1}^{2}\left(X^{2^{n k}}+\alpha^{2^{k}-2} \operatorname{Tr}^{2^{k}}(X)\right)$ which reduced modulo $\left(X^{Q_{+}}\right.$ $X)$ is $C_{k-1}^{2}\left(X+\alpha^{-1}\right)$.

(iii) Using the definition of $A_{\alpha}(X)$ given in Theorem 1,

$$
\begin{aligned}
\operatorname{Tr}\left(A_{\alpha}(X)\right) & =C_{k-1}\left(\operatorname{Tr}(X)^{2^{n k-1}}+\alpha^{\left(2^{k-1}-1\right)} \operatorname{Tr}(X)^{2^{k-1}}\right) \\
& \equiv \operatorname{Tr}(X)^{2^{k-1}} C_{k-1}\left(1+\alpha^{\left(2^{k-1}-1\right)}\right) \bmod \left(X^{Q}+X\right) \\
& \equiv \alpha^{\left(2^{k-1}-1\right)} \operatorname{Tr}(X)^{2^{k-1}} \bmod \left(X^{Q}+X\right) .
\end{aligned}
$$

(iv) This is immediate as $\operatorname{Tr}\left(X \operatorname{Tr}(X)+X^{2}\right)=0$.

The proof of the following result is tedious but seemingly necessary.

PROPOSITION 4. Using the same notation as above then

$$
\begin{aligned}
f_{\alpha}\left(g_{\alpha}(X)\right) \bmod \left(X^{Q}+X\right) & \\
& =X+c_{\alpha}\left(X^{2^{n k-1}} \operatorname{Tr}(X)^{2^{k-1}}+\operatorname{Tr}(X)+\sum_{i=1}^{k} \operatorname{Tr}(X)^{2^{k}-2^{k-i}} S_{i}(X)\right)
\end{aligned}
$$

where $c_{\alpha}=\left(\alpha^{\left(2^{k-1}-1\right)}+1\right) /(\alpha+1)$ and

$$
S_{i}(X)=\sum_{j=1}^{(n-1) / 2}\left(X \operatorname{Tr}(X)+X^{2}\right)^{2^{2 j k-i-1}}
$$

Proof: By expanding $f_{\alpha}(X) \circ g_{\alpha}(X)$ (with $g_{\alpha}(X)=A_{\alpha}(X)+B_{\alpha}(X)$ ) and using Proposition 3 (iv),

$$
\begin{aligned}
& f_{\alpha}\left(g_{\alpha}(X)\right) \bmod \left(X^{Q}+X\right) \\
& \quad=\left(A_{\alpha}(X)+B_{\alpha}(X)\right) \operatorname{Tr}\left(A_{\alpha}(X)\right)+(\alpha+1)\left(A_{\alpha}^{2}(X)+B_{\alpha}^{2}(X)\right) .
\end{aligned}
$$

We split the terms of this sum so that $f_{\alpha}(X) \circ g_{\alpha}(X)=a(X)+b(X) \bmod \left(X^{Q}+X\right)$ where $a(X)=A_{\alpha}(X) \operatorname{Tr}\left(A_{\alpha}(X)\right)+(\alpha+1) A_{\alpha}^{2}(X)$ and $b(X)=B_{\alpha}(X) \operatorname{Tr}\left(A_{\alpha}(X)\right)+(\alpha+1) B_{\alpha}^{2}(X)$.

Using Proposition 3 (ii) and (iii),

$$
\begin{aligned}
a(X)=C_{k-1}^{2}\left(\alpha^{\left(2^{k-1}-1\right)}+1\right)\left(X^{2^{n k-1}}\right. & \left.\operatorname{Tr}(X)^{2^{k-1}}+\alpha^{\left(2^{k-1}-1\right)} \operatorname{Tr}(X)\right) \\
& +C_{k-1}^{2}(\alpha+1)\left(X+\alpha^{-1} \operatorname{Tr}(X)\right) \bmod \left(X^{Q}+X\right) .
\end{aligned}
$$


From Proposition 3 (i), $C_{k-1}^{2}=(\alpha+1)^{-1}$ and as $c_{\alpha}=c_{\alpha} \alpha^{\left(2^{k-1}-1\right)}+\alpha^{-1}$ then

$$
a(X)=X+c_{\alpha}\left(X^{2^{n k-1}} \operatorname{Tr}(X)^{2^{k-1}}+\operatorname{Tr}(X)\right) \bmod \left(X^{Q}+X\right) .
$$

Next put $b_{1}(X)=\operatorname{Tr}\left(A_{\alpha}(X)\right) B_{\alpha}(X)$ : Identically

$$
b_{1}(X)=\alpha^{\left(2^{k-1}-1\right)} \operatorname{Tr}(X)^{2^{k-1}} \sum_{i=1}^{k-1} C_{i} \operatorname{Tr}(X)^{\left(2^{k}-2^{k-1-i}\right)} S_{i+1}(X) .
$$

Using $\alpha^{\left(2^{k-1}-1\right)} C_{i}=\left(\alpha^{\left(2^{k-1}-1\right)}+\alpha^{\left(2^{k-i}-1\right)}\right) /(\alpha+1)$ and re-writing the sum in $b_{1}(X)$ then we arrive at

$$
b_{1}(X)=\sum_{i=2}^{k}\left(\frac{\alpha^{\left(2^{k-1}-1\right)}+\alpha^{\left(2^{k-i}-1\right)}}{\alpha+1}\right) \operatorname{Tr}(X)^{\left(2^{k}-2^{k-i}\right)} S_{i}(X) .
$$

Finally, put $b_{2}(X)=(\alpha+1) B_{\alpha}^{2}(X)$. Then

$$
b_{2}(X)=(\alpha+1) \sum_{i=1}^{k-1} C_{i}^{2} \operatorname{Tr}(X)^{\left(2^{k}-2^{k-1-i}\right)} S_{i+1}^{2}(X) .
$$

As $S_{i+1}^{2}(X)=S_{i}(X)$, from Proposition 3 (i) we have

$$
b_{2}(X)=\sum_{i=1}^{k-1}\left(\frac{\alpha^{\left(2^{k-i}-1\right)}+1}{\alpha+1}\right) \operatorname{Tr}(X)^{\left(2^{k}-2^{k-i}\right)} S_{i}(X) .
$$

So from Equations 2 and 3 we have

$$
\begin{aligned}
b(X) & =b_{1}(X)+b_{2}(X) \\
& =\sum_{i=2}^{k-1} c_{\alpha} \operatorname{Tr}(X)^{\left(2^{k}-2^{k-i}\right)} S_{i}(X)+c_{\alpha} \operatorname{Tr}(X)^{\left(2^{k}-2^{k-1}\right)} S_{1}(X)+c_{\alpha} S_{k}(X) \\
& =c_{\alpha} \sum_{i=1}^{k} \operatorname{Tr}(X)^{\left(2^{k}-2^{k-i}\right)} S_{i}(X) .
\end{aligned}
$$

The result now follows from calculating the sum $a(X)+b(X)$.

Proposition 5. For $\beta \in \mathbb{F}_{q}$ then $f_{\alpha}\left(g_{\alpha}(\beta X)\right)=\beta f_{\alpha}\left(g_{\alpha}(X)\right)$.

Proof: As $\operatorname{Tr}(\beta X)=\beta \operatorname{Tr}(X)$, it is simple to see

$$
(\beta X)^{2^{n k-1}}+\operatorname{Tr}(\beta X)^{2^{k-1}}+\operatorname{Tr}(\beta X)=\beta\left(X^{2^{n k-1}}+\operatorname{Tr}(X)^{2^{k-1}}\right) .
$$

For $\beta \in \mathbb{F}_{q}$, from Equation 1

$$
S_{i}(\beta X)=\sum_{j=1}^{(n-1) / 2} \beta^{2^{2 j k-i}}\left(X \operatorname{Tr}(X)+X^{2}\right)^{2^{2 j k-i-1}}=\beta^{2^{k-i}} S_{i}(X) .
$$


and it follows

$$
\begin{aligned}
\sum_{i=1}^{k} \operatorname{Tr}(\beta X)^{\left(2^{k}-2^{k-i}\right)} \beta^{2^{k-i}} S_{i}(X) & =\sum_{i=1}^{k} \beta^{2^{k}} \operatorname{Tr}(X)^{\left(2^{k}-2^{k-i}\right)} S_{i}(X) \\
& =\beta \sum_{i=1}^{k} \operatorname{Tr}(X)^{\left(2^{k}-2^{k-i}\right)} S_{i}(X) .
\end{aligned}
$$

We then have, using Proposition 4 and these identities, that for $\beta \in \mathbb{F}_{q}, f_{\alpha}\left(g_{\alpha}(\beta X)\right)$ $=\beta f_{\alpha}\left(g_{\alpha}(X)\right)$ as required.

Proof of Theorem 1: For $x \in \mathbb{F}_{Q}$, if $\operatorname{Tr}(x)=0$ then from Proposition 4 it follows directly that $f_{\alpha}\left(g_{\alpha}(x)\right)=x$. Suppose $\operatorname{Tr}(x)=1$ for $x \in \mathbb{F}_{Q}$. Using Proposition 4

$$
\begin{aligned}
f_{\alpha}\left(g_{\alpha}(x)\right) & =x+c_{\alpha}\left(x^{2^{n k-1}}+1+\sum_{i=1}^{k} \sum_{j=1}^{(n-1) / 2}\left(x+x^{2}\right)^{2^{2 j k-1-i}}\right) \\
& =x+c_{\alpha}\left(x^{2^{n k-1}}+1+\sum_{j=1}^{(n-1) / 2} \sum_{i=0}^{k}\left(x+x^{2}\right)^{2^{2 j k-1-i}}\right) \\
& =x+c_{\alpha}\left(x^{2^{n k-1}}+1+\sum_{j=1}^{(n-1) / 2} x^{2^{2 j k}}+x^{2^{(2 j-1) k}}\right) \\
& =x+c_{\alpha}(1+\operatorname{Tr}(x)) .
\end{aligned}
$$

As we have assumed that $\operatorname{Tr}(x)=1$ then again $f_{\alpha}\left(g_{\alpha}(x)\right)=x$. Every element $y \in \mathbb{F}_{Q}$ satisfying $\operatorname{Tr}(y) \neq 0$ can be written in the form $y=\beta x$ where $\beta \in \mathbb{F}_{q}$, and $\operatorname{Tr}(x)=1$ for some $x \in \mathbb{F}_{Q}$. By Proposition $5, f_{\alpha}\left(g_{\alpha}(y)\right)=\beta f_{\alpha}\left(g_{\alpha}(x)\right)=\beta x=y$. Thus $f_{\alpha}\left(g_{\alpha}(X)\right)$ $\equiv X \bmod \left(X^{q}+X\right)$.

The determination of the inverse class given in this article relied on using the MAGMA algebra package [2] to generate examples for small fields. This result underlines that, in general, inverses for known permutation polynomial classes are not simple to describe.

\section{REFERENCES}

[1] A. Blokhuis, R.S. Coulter, M. Henderson and C.M. O'Keefe, 'Permutations amongst the Dembowski-Ostrom polynomials', in Finite Fields and Applications: Proceedings of the Fifth International Conference on Finite Fields and Applications, (D. Jungnickel and H. Niederreiter, Editors) (Springer-Verlag, Berlin, 2001), pp. 37-42.

[2] W. Bosma, J. Cannon and C. Playoust, 'The Magma algebra system I: The user language', J. Symbolic Comput. 24 (1997), 235-265.

[3] R. Lidl and G.L. Mullen, 'When does a polynomial over a finite field permute the elements of the field?', Amer. Math. Monthly 95 (1988), 243-246.

[4] R. Lidl and G.L. Mullen, 'When does a polynomial over a finite field permute the elements of the field?, II', Amer. Math. Monthly 100 (1993), 71-74. 
[5] R. Lidl, G.L. Mullen and G. Turnwald, Dickson Polynomials, Pitman Monographs and Surveys in Pure and Appl. Math. 65 (Longman Scientific and Technical, Essex, England, 1993).

[6] G.L. Mullen, 'Permutation polynomials: a matrix analogue of schur's conjecture and a survey of recent results', Finite Fields Appl. 1 (1995), 242-258.

School of Computing and Mathematics

Deakin University

221 Burwood Highway

Burwood Vic 3123

Australia

e-mail: shrub@deakin.edu.au
Centre for Discrete Mathematics and Computing Department of Computer Science and Electrical Engineering

The University of Queensland

Queensland 4072 .

Australia

e-mail: marie@itee.uq.edu.au 